\title{
R\&D on a new type of micropattern gaseous detector: the Fast Timing Micropattern detector
}

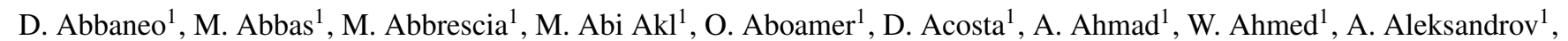
P. Altieri ${ }^{1}$, C. Asawatangtrakuldee ${ }^{1}$, P. Aspell ${ }^{1}$, Y. Assran ${ }^{1}$, I. Awan ${ }^{1}$, S. Bally ${ }^{1}$, Y. Ban ${ }^{1}$, S. Banerjee ${ }^{1}$, V. Barashko ${ }^{1}$, P. Barria ${ }^{1}$, G. Bencze ${ }^{1}$, N. Beni ${ }^{1}$, L. Benussi ${ }^{1}$, V. Bhopatkar ${ }^{1}$, S. Bianco ${ }^{1}$, J. Bos ${ }^{1}$, O. Bouhali ${ }^{1}$, A. Braghieri ${ }^{1}$, S. Braibant $^{1}$, S. Buontempo $^{1}$, C. Calabria ${ }^{1}$, M. Caponero ${ }^{1}$, C. Caputo ${ }^{1}$, F. Cassese ${ }^{1}$, A. Castaneda ${ }^{1}$, S. Cauwenbergh $^{1}$, F.R. Cavallo ${ }^{1}$, A. Celik ${ }^{1}$, M. Choi $^{1}$, S. Choi ${ }^{1}$, J. Christiansen ${ }^{1}$, A. Cimmino ${ }^{1}$, S. Colafranceschi ${ }^{1}$, A. Colaleo ${ }^{1}$, A. Conde Garcia ${ }^{1}$, S. Czellar ${ }^{1}$, M.M. Dabrowski ${ }^{1}$, G. De Lentdecker ${ }^{1}$, R. De Oliveira ${ }^{1}$, G. de Robertis ${ }^{1}$, S. Dildick ${ }^{1,1}$, B. Dorney ${ }^{1}$, G. Endroczi ${ }^{1}$, F. Errico ${ }^{1}$, F. Fallavollita ${ }^{1}$, A. Fenyvesi ${ }^{1}$, S. Ferry ${ }^{1}$, I. Furic ${ }^{1}$, P. Giacomelli ${ }^{1}$, J. Gilmore ${ }^{1}$, V. Golovtsov ${ }^{1}$, L. Guiducci ${ }^{1}$, F. Guilloux ${ }^{1}$, A. Gutierrez ${ }^{1}$, R.M. Hadjiiska ${ }^{1}$, J. Hauser ${ }^{1}$, K. Hoepfner ${ }^{1}$, M. Hohlmann ${ }^{1}$, H. Hoorani ${ }^{1}$, P. Iaydjiev ${ }^{1}$, Y.G. Jeng ${ }^{1}$, T. Kamon ${ }^{1}$, P. Karchin ${ }^{1}$, A. Korytov ${ }^{1}$, S. Krutelyov ${ }^{1}$, A. Kumar ${ }^{1}$, H. Kim ${ }^{1}$, J. Lee ${ }^{1}$, T. Lenzi ${ }^{1}$, L. Litov ${ }^{1}$, F. Loddo ${ }^{1}$, A. Madorsky ${ }^{1}$, T. Maerschalk ${ }^{1}$, M. Maggi ${ }^{1}$, A. Magnani ${ }^{1}$, P.K. Mal ${ }^{1}$, K. Mandal ${ }^{1}$, A. Marchioro ${ }^{1}$, A. Marinov ${ }^{1}$, N. Majumdar ${ }^{1}$, J.A. Merlin ${ }^{1,1}$, G. Mitselmakher ${ }^{1}$,

A.K. Mohanty ${ }^{1}$, A. Mohapatra ${ }^{1}$, J. Molnar ${ }^{1}$, S. Muhammad $^{1}$, S. Mukhopadhyay ${ }^{1}$, M. Naimuddin ${ }^{1}$, S. Nuzzo $^{1}$, E. Oliveri ${ }^{1}$, L.M. Pant ${ }^{1}$, P. Paolucci ${ }^{1}$, I. Park ${ }^{1}$, G. Passeggio ${ }^{1}$, B. Pavlov ${ }^{1}$, B. Philipps ${ }^{1}$, D. Piccolo ${ }^{1}$, H. Postema ${ }^{1}$, A. Puig Baranac ${ }^{1}$, A. Radi ${ }^{1}$, R. Radogna ${ }^{1}$, G. Raffone ${ }^{1}$, A. Ranieri ${ }^{1}$, G. Rashevski ${ }^{1}$, M. Ressegotti ${ }^{1}$, C. Riccardi ${ }^{1}$, M. Rodozov ${ }^{1}$, A. Rodrigues ${ }^{1}$,

L. Ropelewski ${ }^{1}$, S. RoyChowdhury ${ }^{1}$, G. Ryu ${ }^{1}$, M.S. Ryu ${ }^{1}$, A. Safonov ${ }^{1}$, S. Salva ${ }^{1}$, G. Saviano ${ }^{1}$, A. Sharma ${ }^{1}$, A. Sharma $^{1}$, R. Sharma ${ }^{1}$, A.H. Shah ${ }^{1}$, M. Shopova ${ }^{1}$, J. Sturdy ${ }^{1}$, G. Sultanov ${ }^{1}$, S.K. Swain ${ }^{1}$, Z. Szillasi ${ }^{1}$, J. Talvitie ${ }^{1}$, A. Tatarinov ${ }^{1}$, T. Tuuva ${ }^{1}$, M. Tytgat ${ }^{1}$, I. Vai ${ }^{1}$, M. Van Stenis ${ }^{1}$, R. Venditti ${ }^{1}$, E. Verhagen ${ }^{1}$, P. Verwilligen ${ }^{1}$, P. Vitulo ${ }^{1}$, S. Volkov $^{1}$, A. Vorobyev ${ }^{1}$, D. Wang ${ }^{1}$, M. Wang ${ }^{1}$, U. Yang ${ }^{1}$, Y. Yang ${ }^{1}$, R. Yonamine ${ }^{1}$, N. Zaganidis ${ }^{1}$, F. Zenoni ${ }^{1}$, A. Zhang ${ }^{1}$

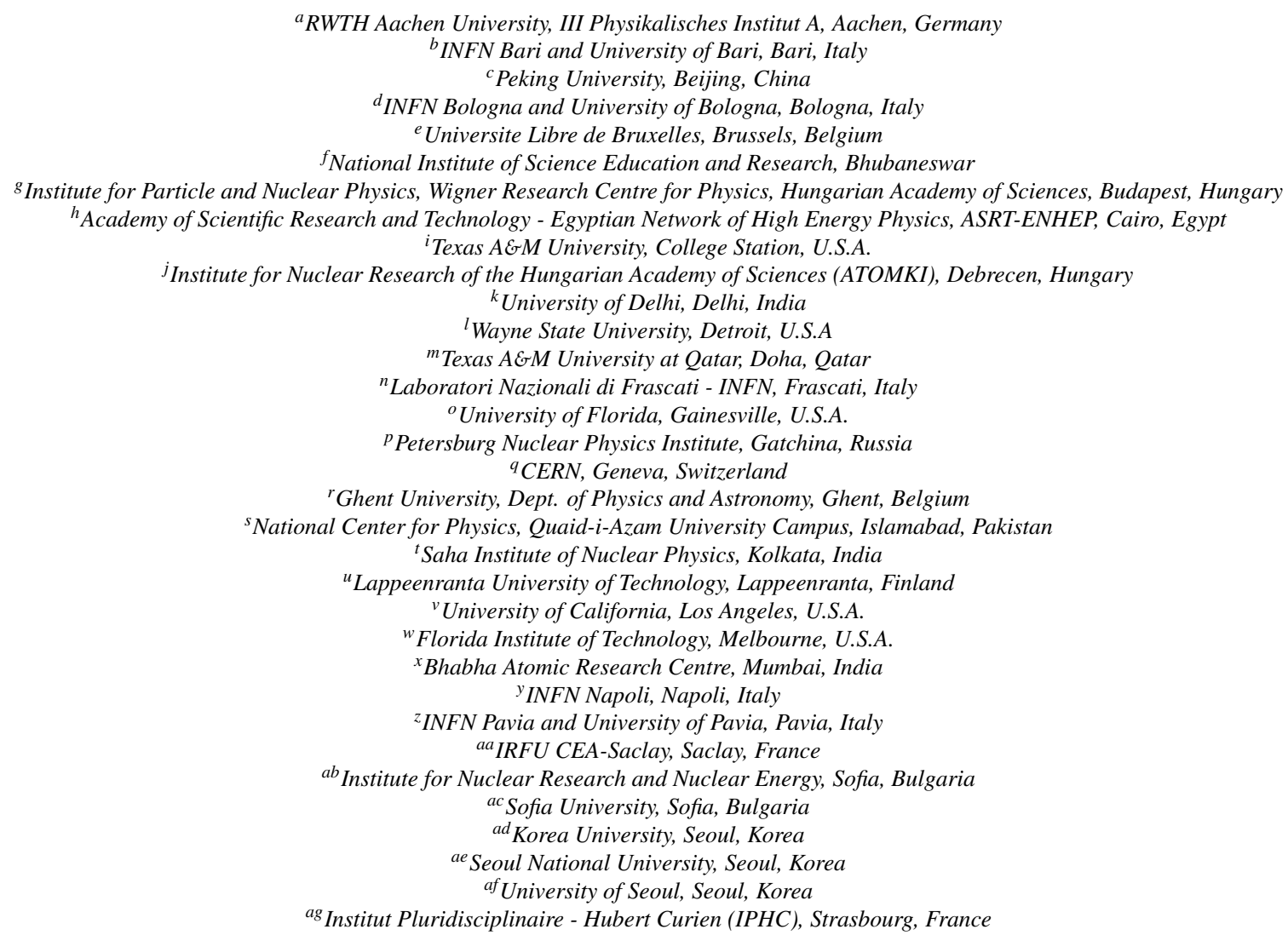

\section{Abstract}

This contribution introduces a new type of Micropattern Gaseous Detector, the Fast Timing Micropattern (FTM) detector, utilizing fully Resistive WELL structures. The structure of the prototype will be described in detail and the results of the characterization study performed with an X-ray gun will be presented, together with the first results on time resolution based on data collected with muon/pion test beams. 


\section{Introduction}

Time resolution of classical Micropattern Gas Detectors ${ }_{38}$ (MPGD), like Gas Electron Multiplier (GEM) and Micromegas, is dominated by the fluctuations on the position on the first ion- ${ }_{40}$ ization cluster in the drift gap. The average time needed for the ${ }_{41}$ nearest ionization cluster to reach the amplification stage is in- ${ }_{42}$ deed given by $t=d / v_{d}$, where $d$ is the distance of the closest ${ }_{43}$ cluster to the first amplification region and follows the distri- ${ }_{44}$ bution $\mathrm{e}^{-\lambda x} / \lambda$, where $\lambda$ is the average number of primary clusters ${ }_{45}$ generated by an ionizing particle inside the gas per length; $v_{d}$ is the drift velocity, that depends on the gas mixture and the applied drift field. The contribution to the time resolution of the drift velocity is $\sigma_{t}=\left(\lambda v_{d}\right)^{-1}$ : with a typical drift gap of the ${ }^{47}$ order of 3-4 $\mathrm{mm}$ and with a proper choice of the gas mixture, MPGDs can reach a time resolution of the order of 5-10 ns. An ${ }^{48}$ improvement in the time resolution, to reach the $1 \mathrm{~ns}$ scale, can ${ }^{49}$ be obtained working on the segmentation of the drift gap: the principle is to divide a single thick drift region in many thin- ${ }^{5}$ ner drift regions, each coupled to its amplification stage. The reduction in time resolution that can be obtained is so proportional to the number of stages $N_{D}$ employed: $\sigma_{t}=\left(\lambda v_{d} N_{D}\right)^{-1}$. The first prototype of Fast Timing Micropattern (FTM) detector exploits this principle using two $250 \mu \mathrm{m}$-thick drift gaps, each coupled with an amplification region composed by a fully resistive WELL. The construction of consecutive drift-amplification stages is allowed by the use of resistive layers to polarize drift and multiplication volumes. The overall structure is then transparent to the signal that can be extracted from every amplification stage.

\section{The Fast Timing Micropattern detector}

The structure of the first prototype of Fast Timing Micropattern (FTM) detector is described in [? ]. It is composed of two

foil

8 $800 \mathrm{M} \Omega$; with a thick, with planarity ensured by overlay pillars, $400 \mu \mathrm{m}$ diameter and pitch of $3.3 \mathrm{~mm}$. The active area of the prototype is of the order of $20 \mathrm{~cm}^{2}$. The induced signal can be picked up from the readout electrode, but also from the drift electrode, through a capacitive coupling.

\section{Characterization with $X$-Rays}

The first characterization of the FTM prototype was performed at CERN with a AMPTEK Mini-X X-ray tube, with Ag cathode filament ( $22 \mathrm{keV} X$-Rays). Examples of signals picked up from the drift and readout electrodes and read out with an electronics chain composed by a preamplifier ORTEC 142PC and an amplifier ORTEC 474, are shown in Fig. ??.

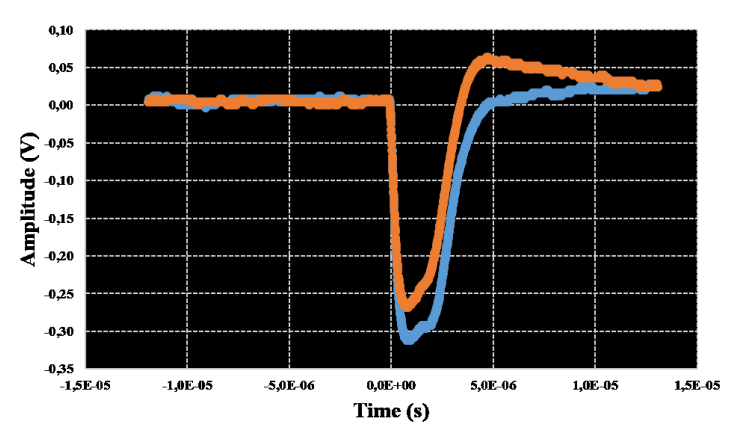

Figure 2: Signals from the FTM detector after amplification and shaping made with a preamplifier ORTEC 142PC and an amplifier ORTEC 474: in blue the signal pickup from the readout electrode, in orange from the drift electrode through a capacitive coupling, the latter being inverted.

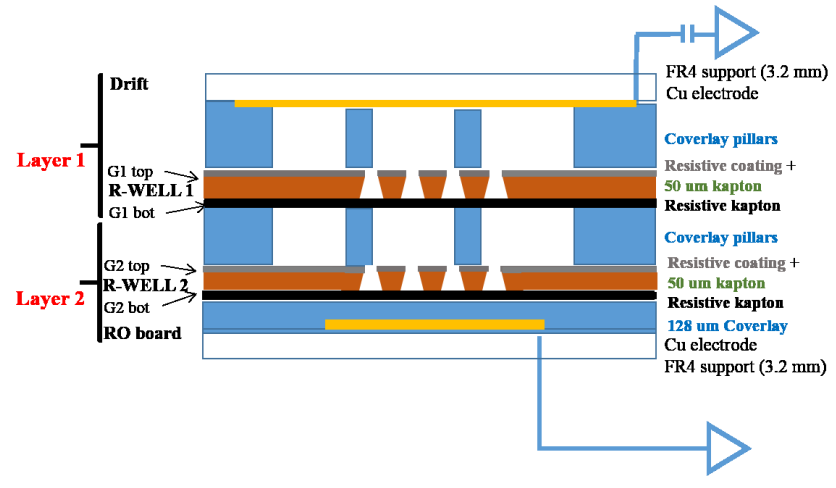

Figure 1: Transverse view of the first prototype of FTM detector.

independent drift-amplification stages (Fig. ??): each amplifi- 65 cation region is based on a pair of polyimide foils, i.e. kap- ${ }^{66}$ ton, stacked due to the electrostatic force induced by the po- ${ }^{67}$ larization of the foils: the first foil, perforated with inverted ${ }^{68}$

${ }^{*}$ Corresponding author
The rate from both the readout and drift electrodes at different values of current from the X-Ray gun, i.e different values of incident flux up to the maximum available from the source, is shown in Fig. ??. The response of the detector, for both the electrodes, is linear; in addition the two data sets are comparable, giving an indication of the electrical transparency of the layers.

\section{Time resolution measurement}

A two-week test beam was carried out in autumn 2015 at the SPS H4 beam line [? ] at CERN, with muon and pion beams, with the aim of measuring the time resolution of the detector. The setup used during the test and shown in Fig. ?? was instrumented with three $10 \times 10 \mathrm{~cm}^{2}$ Triple-GEM detectors, 3:2:2:2 mm gap configuration, for alignment with the beam and four scintillators, including one $2.5 \times 3.5 \mathrm{~cm}^{2}$ finger scintillator, for triggering.

The time resolution was evaluated with muon and pion beams in different powering configuration of the detector. 


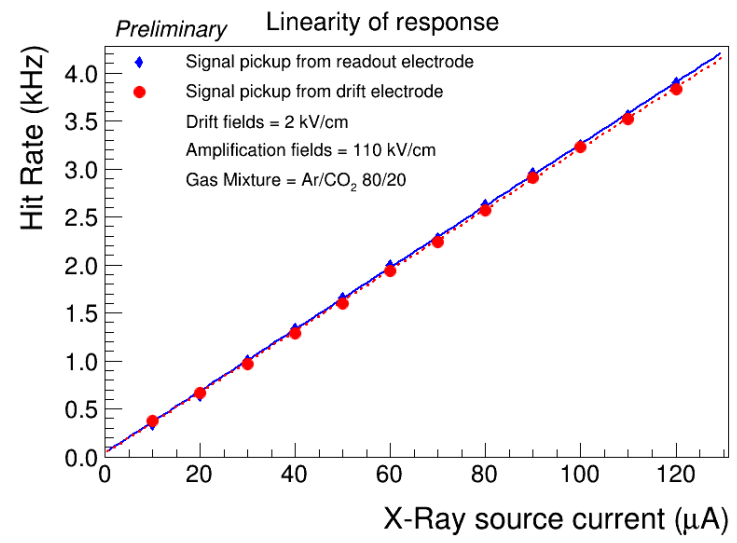

Figure 3: Measured hit rate from both channels of the FTM detector as a function of the X-ray current, which is proportional to the incident flux, up to the maximum available from the source. The increase of the measured rate is linear with the increase of the incident flux. In addition, the data sets from readout (blue diamonds, full line) and drift (red points, dashed line) channels are comparable, outlining the electrical transparency of the layers.

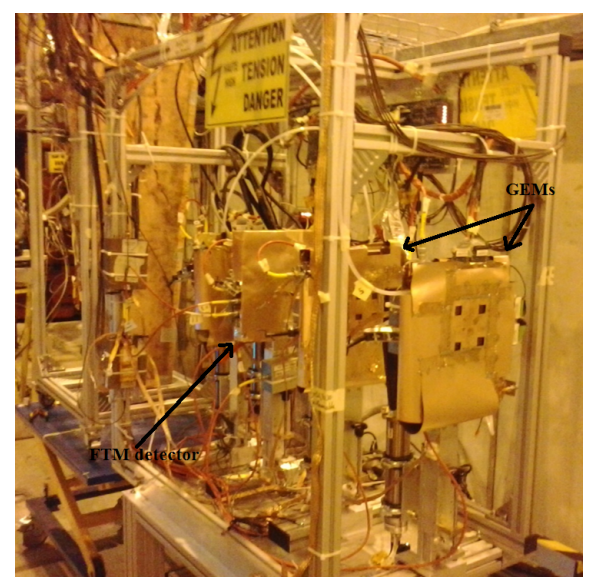

Figure 4: Stand used for the time resolution measurement during 2015 test beam: from the front, two $10 \times 10 \mathrm{~cm}^{2}$ Triple-GEM, the FTM detector with the squared copper case and the last Triple-GEM for alignment. The four scintilla- 88 tors are placed behind each detector, in particular the finger scintillator is placed just behind the FTM detector in order to match the active area of the prototype and improve the geometrical acceptance of the trigger.

Fig. ?? shows the time distribution of events induced by 93 13 muons: the signal is taken from the drift electrode and read 94 14 out by a fast electronic chain composed by a CIVIDEc broadband 95 amplifier and a linear Lecroy 612AM amplifier. The time res- 96 olution is the sigma of the gaussian fit to the time distribution 97 7 and is of the order of $2.4 \mathrm{~ns}$. The same result obtained with pi- 98 78 ons is shown in Fig. ??: here the time resolution is of the order 99 of $1.7 \mathrm{~ns}$. All these results were achieved using a green-house-100 gas-free gas mixture, composed by $\mathrm{Ar} / \mathrm{CO}_{2} 70 / 30$.

Fig. ?? shows the measured time resolution for different values of applied drift fields, keeping constant the amplification ${ }_{102}$

fields, with both muon and pion beams. The time resolution of ${ }_{103}$ the detector seems is not affected by a change in drift field: this is due to the fact that, with such a small drift gap, even a big ${ }_{104}$ change in the drift velocity would not affect significantly the ${ }_{105}$ time needed by electrons to reach the amplification region. $\operatorname{In}_{106}$

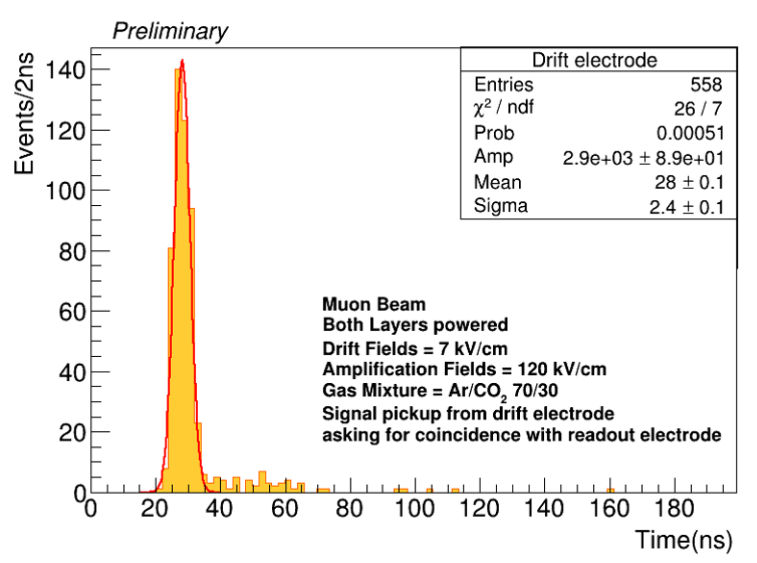

Figure 5: Detector time distribution of the events induced by muons during 2015 test beam. The time resolution was evaluated from the sigma of the gaussian fit.

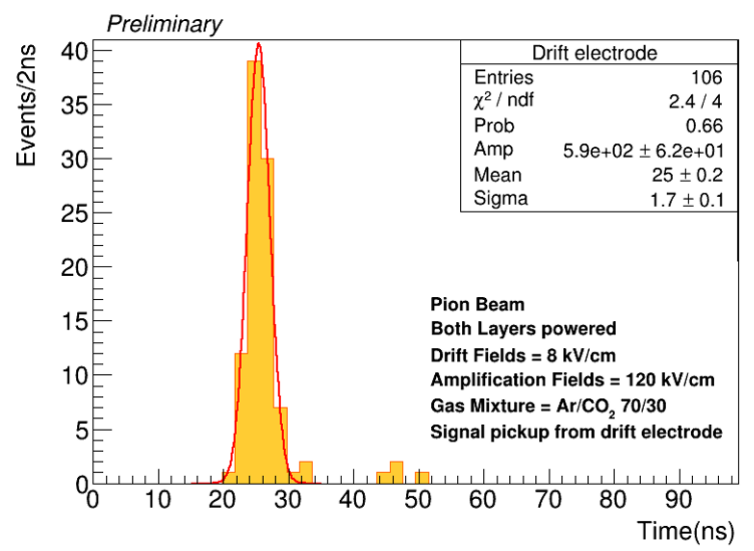

Figure 6: Detector time distribution of the events induced by pions during 2015 test beam. The time resolution was evaluated from the sigma of the gaussian fit.

addition, the drift velocity in $\mathrm{Ar} / \mathrm{CO}_{2} 70 / 30$ mixture is nearly constant, with variations of the order of $5 \%$ for drift fields between 2 and $10 \mathrm{kV} / \mathrm{cm} \mathrm{[?].}$

Recalling the formula $\sigma_{t}=\left(\lambda v_{d} N_{D}\right)^{-1}$ we can make a rough estimation of the time resolution expected for an efficient twolayer detector: assuming $\lambda \sim 33 \mathrm{~cm}^{-1}$ for $\mathrm{MIP}$ in $\mathrm{Ar} / \mathrm{CO}_{2}$ $70 / 30$ mixture, $v_{\mathrm{d}} \sim 8 \mathrm{~cm} / \mu \mathrm{s}$, the estimated time resolution is $\sigma_{\mathrm{t}} \sim 1.9 \mathrm{~ns}$. The actual measurement of time resolution in this prototype is affected by two competing phenomena: first, the prototype is extremely thin so that some of the traversing particles will not ionize in the drift volume, which truncates the tail of the timing distribution. On the other hand, the measurement accuracy is limited by the TDC resolution and the sensitivity of the front-end electronics.

\section{Application in High Energy Physics experiments: the CMS muon system in the high $\eta$ region}

With the improvement in luminosity foreseen for HighLuminosity LHC, the flux of particles through the CMS detector will greatly exceed that in previous running. Keeping the 


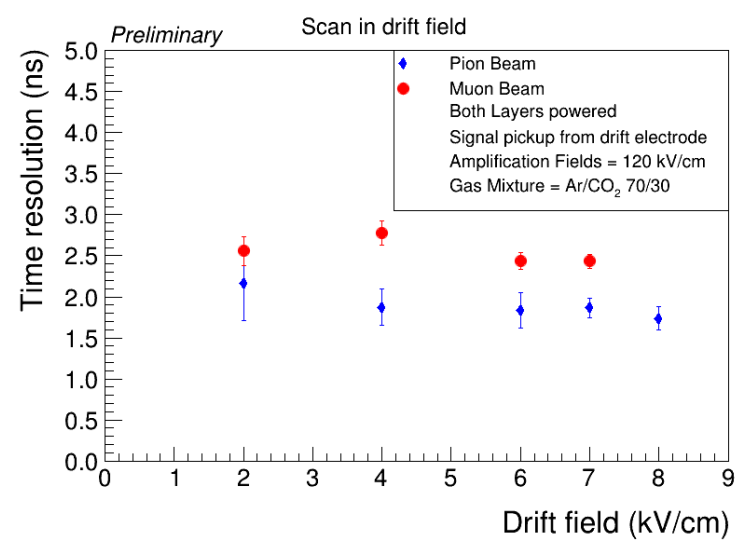

Figure 7: Measured time resolution for different values of applied drift field: ${ }^{140}$ the red points refer to muon beam, the blue diamonds to pion beam.

same performance of Run 1 will be a great challenge for the 143 collaboration. In particular, focusing on the high pseudorapid-144 ity $(\eta)$ region of the muon system of the CMS experiment the 145 biggest challenges will be:

- Redundancy: the high $\eta$ region is the region with the high- ${ }^{147}$ est rates but the fewest muon layers;

- Rate: increases towards higher $\eta$;

- Detector Longevity: after years of LHC operation the ac-150 cumulated charge will reach values of the order of few ${ }^{15}$ $\mathrm{C} / \mathrm{cm}^{2}$;

- Electronics: the existing electronics is not able to handle ${ }_{154}$ the occupancies/rates and latencies.

In order to face this conditions the CMS Collaboration de- ${ }^{156}$ cided to improve the muon system, also with the introduction ${ }^{157}$ of new stations instrumented with MPGDs, as shown in Fig. ?? ${ }_{159}$ The GEM Endcap 1/1 (GE1/1) station will be instrumented with GEM detectors and installed during Long Shutdown 2 (LS2) [? ]; for the other two stations proposed for LS3, GE2/1 and ME0 (Muon Endcap 0), the baseline solution is again GEM detec-161 tors, but optional solutions foresee $\mu$ RWELL technology [?] for GE2/1 and the FTM technology for ME0.

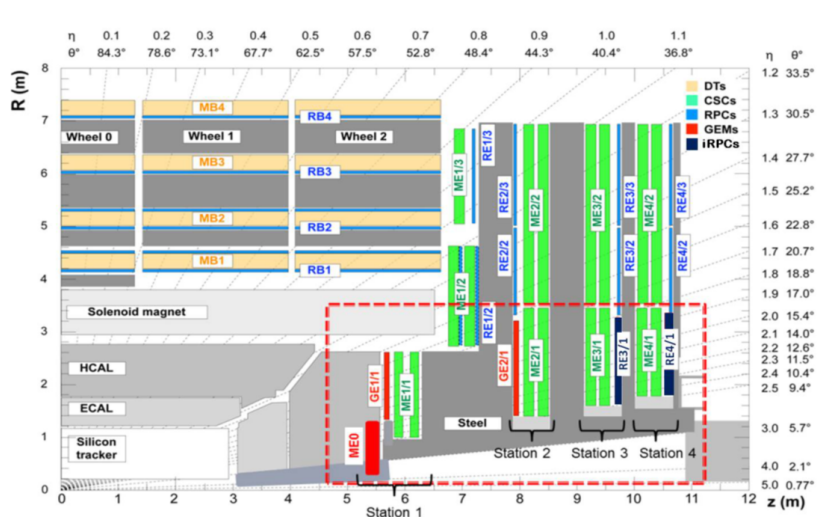

Figure 8: The CMS muon system instrumented with MPGD.
The purpose of the ME0 station in particular is the increase in pseudorapidity coverage up to $|\eta|<3$. In addition, in order to match with the new tracker that will provide triggering up to $|\eta|<2.4$, the ME0 station should also provide a robust muon trigger with low $\mathrm{p}_{T}$ threshold and muon tagging. The conditions in which the station would have to operate will be extremely harsh, with a pile-up of the order of 140-200 and a very high background rate up to $100 \mathrm{kHz} / \mathrm{cm}^{2}$. For these reasons the detectors to be installed in the proposed station will need to have:

- High granularity and spatial segmentation, to allow $\mathrm{p}_{T}$ assignment and improve pile-up rejection;

- Multi-layered structure, to allow an improvement of local muon track reconstruction and discrimination between muons (resulting in a segment) and neutrons (resulting in uncorrelated hits);

- Timing, for good track reconstruction, reduction of in-time pile up and help in vertex association. Also neutron background mitigation will benefit from timing: if the detection location is known precisely, only small time windows are compatible with genuine muon hits from the interaction point.

\section{Summary}

The first prototype of Fast Timing Micropattern detector was tested and proved to have a linear response to the rate, to be electrically transparent and its time resolution was measured to be of the order of 1.5 - $2.5 \mathrm{~ns}$ with $\mathrm{Ar} / \mathrm{CO}_{2}$ 70/30 gas mixture.

The detector is being considered for applications in high energy physics experiments, including the CMS muon system.

The R\&D is ongoing with the design of a new prototype, fully PCB-based, with at least 4 independent stages, that will be tested at the SPS test beam facility and at the Gamma Irradiation Facility $(\mathrm{GIF}++)[?]$ at CERN in presence of high gamma-ray background.

\section{Acknowledgments}

We gratefully acknowledge the support of FRS-FNRS ${ }_{63}$ (Belgium), FWO-Flanders (Belgium), BSF-MES (Bulgaria),
BMBF (Germany), DAE (India), DST (India), INFN (Italy), NRF (Korea), QNRF (Qatar), and DOE (USA).

\section{References}

[1] R. De Oliveira et al., A novel fast timing micropattern gaseous detector: FTM, arXiv:1503.05330.

[2] SPS infrastucture, http://ab-dep-op-sps.web.cern.ch/ab-dep-op-sps/.

[3] Y. Assran et al., Transport properties of operational gas mixtures used at LHC, arXiv:1110.6761.

[4] CMS GEM Collaboration, CERN-LHCC-2015-012.

[5] G.Bencivenni et al., The micro-Resistive WELL detector: a compact spark-protected single amplification-stage MPGD, 2015 JINST 10 P02008.

[6] GIF++, https://espace.cern.ch/sba-workspace/gifpp/SitePages/Home.aspx 